\title{
IMPACT OF GROWTH PROMOTERS ADDITION ON PRODUCTIVE PERFORMANCE AND IMMUNE RESPONSE IN BROILER CHICKENS
}

\author{
Hashim $^{1^{\star}}$ M.K.M., El-Shinawy ${ }^{2}$ M.Z., Mahrous ${ }^{3}$ M.Y. and Galal ${ }^{3}$ A. \\ 1- Arid Soils Dept., Fac. of Agric., Ain Shams Univ., P.O. Box 68, Hadayek Shoubra 11241, \\ Cairo, Egypt. \\ 2- Horticulture Dept., Fac. of Agric., Ain Shams Univ., P.O. Box 68, Hadayek Shoubra 11241, \\ Cairo, Egypt. \\ 3- Poultry Dept., Fac. of Agric., Ain Shams Univ., P.O. Box 68, Hadayek Shoubra 11241, Cairo, \\ Egypt.
}

*Corresponding author: mohamedhashem10@icoud.com

Received 11 September, 2019

Accepted 12 January, 2020

\section{ABSTRACT}

The purpose of this work was to study the effect of Fenugreek seeds powder (Trigonella foenum-gracum L.) and Camphor leaves powder (Cinnamomum camphora) on productive performance and immune response of Ross broiler chicken. A total of 100 day-old unsexed broiler chicks (Ross 308 ) were randomly allocated to five treatment groups with two replicates of 10 each reared for 42 days. The experimental diets were: Control diet $(C)$, fenugreek seeds powder $1 \mathrm{~g} / 1 \mathrm{~kg}$ (F1), fenugreek seeds powder $1.5 \mathrm{~g} / 1 \mathrm{~kg}(\mathrm{~F} 2)$, camphor leaves powder $1 \mathrm{~g} / 1 \mathrm{~kg}$ (Cph1), camphor leaves powder $1.5 \mathrm{~g} / 1 \mathrm{~kg}$ (Cph2). The F2 trait recorded the best results during most of ages for body weight and body weight gain. Dressing percentage of broilers at five week was significantly higher value for Cph1 group. The percentage of minor and major breast muscles showed a slightly nonsignificant increase to Cph2 trait. The drum muscle weight percentage showed a higher significant value for (Cph1) trait. The F2 group recorded the lower significant value for Giblets percentage compared to another groups and control group. The highest value for spleen percentage was recorded in F1 group and the lowest value showed in control and Cph2. Bursa gland recorded the highest value for F2 group and the lowest value recorded by Control and Cph2 group. The F1 group recorded the highest value for thymus gland percentage and the lowest value showed in Cph2 group. The Cph2 and F2 treatments had the maximum cutaneous basophilic hypersensitivity swelling response with insignificantly difference at $24 \mathrm{hr}$ after injection, while at $48 \mathrm{hr}$, the Cph2 trait had significantly higher dermal swelling response compared to other traits and control group. The F1 and F2 groups had significantly hyper responder to sheep red blood cells at seven days post-secondary injection, while at fourteen days post-secondary injection, Cph2 and F2 groups had significantly hyper responses to sheep red blood cells. Therefore, fenugreek seeds and camphor leaves supplementation as a growth promoter at $1.5 \mathrm{~g} / 1 \mathrm{~kg}$ might be acceptable for achieving better performance under environmental conditions of Egypt.

Keywords: Fenugreek, Camphor, Productive, Immune, Broiler

\section{INTRODUCTION}

Antibiotics as growth promoter in chicken diets are considered serious health risks to human health, because of their residual effects in meat result pathogens develop resistance to antibiotics. Scientists of poultry today are challenged to find out new alternatives to antibiotic growth promoters with no side effects for poultry that could be more or as effective against harmful micro-organisms and to stimulate the growth by increasing the efficiency of feed utilization and to enhance the immunity (Farman et al 2009). The development of resistance to certain antibiotics poses real problems to the animal and public health (Barton, 2000). Consequently, many additives (prebiotics, probiotics, symbiotic....) raise a particular interest as products of substitution to antibiotics in order to improve the production performances and the health of animals (Bach, 2001). 
Fenugreek leaves and seeds have been used extensively to prepare extracts and powders for medicinal (Basch et al 2003). Fenugreek has been used for over two thousand years as a medicinal plant in various parts of the world (Srinivasan, 2006). Use of fenugreek is associated with a wide range of therapeutic applications including a carminative (prevents flatulence) and as an aphrodisiac. Fenugreek seeds are considered as an appetizer and helps indigestion. The seed has antioxidant, antiviral and anticarcinogenic activities (Mazur et al 1998). The plant is often used for over two thousand years as a medicinal plant in various parts of the world.

Camphor, a natural product derived from the wood of the tree Cinnamomum camphora, has a longhistory use as antiseptic, analgesic, antipruritic, counterirritant and rubefacient (Ellenhorn and barceloux, 1998). It success and wide medical use, especially in topical preparations, is connected to its mild local an esthesizing effect and to the production of a circum scribed sensation of heat, together with its characteristic and penetrating odour that is by most of people associated to the idea of a strong and effective medicine (Gibson et al 1989). Then, we study the effect of fenugreek seeds and camphor leaves powder on productive performance and immune response of Ross broiler chicken.

\section{MATERIALS AND METHODS}

\section{Study area}

The experimental work of this study was carried out at the poultry-Breeding farm, Poultry Production Department, Faculty of Agriculture, AinShams University, during the period from August to September 2015. One-day old chicks from Ross commercial broiler strain has been reared to 6 weeks-old of age.

\section{Preparation of the Experimental Diets}

Two medicinal plants, namely Trigonella foenum-graecum L. (fenugreek) and Camphor (Cinnamomum camphora) was purchased from the local area of Giza governorate, Egypt. The medicinal plants were washed with cold water and dried under shade. The dried leaves of Camphor and fenugreek seeds were coarsely powdered. The samples were further ground into powder. The obtained powder was packed and preserved in the feed storage room until used for feed formulation. Based on the chemical analysis result, two treatment rations containing fenugreek at levels of $(1 \%$ and $1.5 \%)$ and another two treatment rations containing camphor at levels of ( $1 \%$ and $1.5 \%)$ of the total ration were formulated in addition to control diet with two replicates for every treatment as: Control diet $(\mathrm{C})$, fenugreek seeds powder $1 \mathrm{~g} / \mathrm{kg}$ diet (F1), fenugreek seeds powder $1.5 \mathrm{~g} / 1 \mathrm{~kg}(\mathrm{~F} 2)$, camphor leaves powder $1 \mathrm{~g} / 1 \mathrm{~kg}$ (C1), camphor leaves powder $1.5 \mathrm{~g} / 1 \mathrm{~kg}(\mathrm{C} 2)$. The rations were formulated to be with Metabolizable Energy (ME) content of $3000 \mathrm{kcal} / \mathrm{kg}$ Dry Matter (DM) and Crude Protein (CP) content of $23 \%$ during the starter phase of 1 to 17 days of age, ME content of 3050 $\mathrm{kcal} / \mathrm{kg} \mathrm{DM}$ and CP content of $21 \%$ for grower phase of 18 to 29 days of age and ME content of $3100 \mathrm{kcal} / \mathrm{kg} \mathrm{DM}$ and CP content of $19 \%$ for finisher phase of 30 to 42 days of age. The diets introduced for chickens ad libitum.

\section{Measurements}

All birds have been individually weighed weekly intervals (at 0, 1, 2, 3, 4 and 5 wk of ages). Also, body weight gain was estimated weekly from $0-1$, 1-2, 2-3, 3-4 and 4-5 week of age and calculated for accumulative period of 0-5 week of age.

At $6^{\text {th }}$ week of age, 5 birds from each treatment were slaughtered for carcass evaluation. Edible and inedible parts were extracted and weighed. The lymphoid organs (Bursa of Fabricius, Spleen and Thymus) of these slaughtered birds have been extracted and weighed also.

Response induced in vivo by mitogen was evaluated by injection of phytohemagglutinin- $P$ (PHA-P) into the toe webs between the second and the third digits of chicks. Five birds from each treatment at 4 weeks of age were used. Each bird was intradermally injected in the toe web of the left foot with $100 \mu \mathrm{g}$ phytohemagglutinin-P (Sigma Chemical Co., St. Louis, MO 63178) in $0.1 \mathrm{ml}$ of sterile saline measured with a constant tension caliper before injection and at 24, 48 and $72 \mathrm{hr}$ after PHA-P injection. The toe web swelling was calculated as the difference between the thickness of the toe web before and after injection.

Sheep red blood cells (SRBCs) were used as T-dependent antigens to quantify the antibody response. Five birds from each trait were injected intramuscular with SRBCs (3\% suspension in PBS, $1 \mathrm{ml} /$ bird) at 2 weeks of age followed by a booster injection of SRBC suspension at 4 weeks (after 14 days of the first injection). Blood samples were collected at 7 and 14 days after the first injection and again at 7 and 14 days post booster. The plasma from each sample was collected; heat inactivated at $56{ }^{\circ} \mathrm{C}$ for $30 \mathrm{~min}$ and then analyzed for 

response in broiler chicken

total immunoglobulin, mercaptoethanol-sensitive (MES) and mercaptoethanolresistant (MER) IgG anti-SRBC antibodies as previously described (Yamamoto and Glick, 1982). Briefly, $50 \mu \mathrm{L}$ of plasma was added in an equal amount of PBS in the first column of a 96-well V-shaped bottom plate, and the solution was incubated for $30 \mathrm{~min}$ at $37^{\circ} \mathrm{C}$. A serial dilution was then made (1:2) and 50 $\mu \mathrm{L}$ of $2 \%$ SRBC suspension was added to each well. Total antibody titers were then read after 30 min. of incubation at $37^{\circ} \mathrm{C}$. The well immediately preceding a well with a distinct SRBC button was considered as the endpoint titer for agglutination. For MES (IgM) response, $50 \mu \mathrm{L}$ of $0.01 \mathrm{M}$ mercaptoethanol in PBC was used instead of PBS alone, followed by the aforementioned procedure. The difference between the total and the IgG response was considered to be equal to the $\operatorname{lgM}$ antibody level.

\section{Statistical analysis}

Data were subjected to a one-way analysis of variance with treatments effect using the General Linear Model (GLM) procedure of (SAS User's Guide 9.1.3, 2004) using the following model:

$$
Y i j=\mu+S i+e i j
$$

Where; Yij = Trait measured, $\mu=$ Overall means, $\mathrm{Si}=$ Additional of growth promoters, eij $=$ Experimental error.

Duncan's multiple range test (Duncan, 1955) was used to separate means when the dietary treatments effect was significant.

\section{RESULTS AND DISCUSSION}

\section{Productive performance}

\subsection{Body weight}

Data presented in Table (1) showed a significant difference between traits at 4 week of age. Chicks which fed on (Camphor 2) showed increase in body weight at 4 week (1351.73 g) compared to other traits. While (F1 \& F2) traits recorded nonsignificant increase in body weight (1628.55 \& $1631.25 \mathrm{~g}$ ) respectively, compared to other groups. The (F2) trait recorded the best body weight during most of ages.

In contrast to our findings, Weerasingha et al (2013) concluded that $1 \%$ dietary fenugreek seed powder has some growth promoting effects in broilers, while higher than $1 \%$ dietary fenugreek has negative effects on feed intake and growth performance. Mamoun et al (2014) investigated chicks fed on $1 \%$ fenugreek seed powder recorded significantly the higher body weight compared to all tested groups, while those fed on control diet recorded significantly the lowest body weight value.

\subsection{Body weight gain}

The presented data in Table (2) illustrated that there is no effect to camphor and fenugreek addition into dietary on body weight gain. The control group recorded a higher significant value than traits which fed on dietary containing camphor and fenugreek additives. A cumulative value of body weight gain during the age between 0-5 weeks recorded higher value (1556.06 g) to control group than other groups. The (F2) group showed the higher value $(1388.89 \mathrm{~g})$ for cumulative body weight gain after the control group. While, Khadr and Abdel-Fattah (2007) indicated that addition of fenugreek seeds during the growing period had slightly increased body weight gain for chicks fed diets containing $1 \%$ fenugreek seeds followed by those fed $2 \%$ level, especially in the last week of the experimental period.

\subsection{Edible parts}

In the current study, Table (3) showed that dressing percentage of broilers at 5 week was significantly higher value for Cph1 group (75.14\%) than control $(73.20 \%)$ and other groups. The percentage of minor and major breast muscles showed a non-significant increase to Cph2 trait (2.44\% \& 9.18\% subsequently) compared to control and other treatments F2 group recorded the lower significant value for Giblets percentage $(3.43 \%)$ compared to another groups and control group. Drum muscle weight percentage showed a higher significant value for (Cph1) trait which recorded $(5.05 \%)$, followed by (F1) treatment which recorded $(4.80 \%)$. While, control recorded $(4.29 \%)$ for drum weight percentage. The (F2) treatment had a lower value of both drum and thigh muscles percentages as follow $(4.21 \mathrm{~g}$ and $6.55 \mathrm{~g})$ subsequently compared to control group.

Khadr and Abdel-Fattah (2007) showed that broilers fed diets supplemented with $1 \%$ or $2 \%$ fenugreek seeds decreased percentages of dressing, heart and edible organs but the differences were insignificant compared to the control group. Feeding $3 \mathrm{~g} / \mathrm{kg}$ of fenugreek seeds insignificantly affected all slaughters parameters (Rabia, 2010). 
Table 1. Weekly body weight of Ross broiler hybrids affected by Camphor and Fenugreek

\begin{tabular}{|c|c|c|c|c|c|c|}
\hline Age (wk.) & Control & Cph1 & Cph2 & F1 & F2 & Pr. \\
\hline $\mathbf{0}$ & $42.01 \pm 1.37$ & $41.89 \pm 2.11$ & $40.88 \pm 1.88$ & $43.24 \pm 1.97$ & $42.36 \pm 0.94$ & NS \\
$\mathbf{1}$ & $152.00 \pm 2.98$ & $155.59 \pm 3.27$ & $154.74 \pm 3.17$ & $152.88 \pm 3.42$ & $152.80 \pm 2.92$ & NS \\
$\mathbf{2}$ & $397.52 \pm 14.61$ & $401.83 \pm 16.18$ & $414.87 \pm 9.64$ & $404.06 \pm 7.83$ & $423.06 \pm 11.16$ & NS \\
$\mathbf{3}$ & $768.80 \pm 20$ & $806.81 \pm 27.42$ & $806.58 \pm 16.51$ & $831.05 \pm 16.30$ & $836.87 \pm 15.60$ & NS \\
$\mathbf{4}$ & $1270.67^{\mathrm{ab}} \pm 15.76$ & $1204.92^{\mathrm{b}} \pm 34.23$ & $1351.73^{\mathrm{a}} \pm 39.52$ & $1263.40^{\mathrm{ab}} \pm 33.02$ & $1284.64^{\mathrm{ab}} \pm 22.95$ & 0.031 \\
$\mathbf{5}$ & $1611.25^{\mathrm{a}} \pm 47.86$ & $1567.82^{\mathrm{b}} \pm 61.43$ & $1583.33^{\mathrm{b}} \pm 18.42$ & $1628.55^{\mathrm{a}} \pm 33.03$ & $1631.25^{\mathrm{a}} \pm 34.93$ & 0.048 \\
\hline
\end{tabular}

$\mathrm{a}$ and $\mathrm{b}$ Means within the same row with different letters are significantly differed, $\mathrm{C}=\mathrm{Control}$, Cph1=Camphor 1, Cph2=Camphor 2, F1=Fenugreek 1, F2=Fenugreek 2, Pr.= probability, NS = non-significant.

Table 2. Weekly and cumulative body weight gain of Ross broiler hybrids affected by Camphor and Fenugreek

\begin{tabular}{|c|c|c|c|c|c|c|}
\hline Age (wk.) & Control & Cph1 & Cph2 & F1 & F2 & Pr. \\
\hline $\mathbf{0 - 1}$ & $109.99 \pm 6.88$ & $113.70 \pm 8.39$ & $113.86 \pm 8.17$ & $109.64 \pm 6.74$ & $110.44 \pm 7.45$ & NS \\
$\mathbf{1 - 2}$ & $229.95 \pm 14.75$ & $260.78 \pm 9.70$ & $248.95 \pm 12.35$ & $246.60 \pm 8.75$ & $257.90 \pm 14.35$ & NS \\
$\mathbf{2 - 3}$ & $406.10 \pm 34.28$ & $391.00 \pm 23.28$ & $364.41 \pm 27.90$ & $425.40 \pm 20.00$ & $406.42 \pm 2.14$ & NS \\
$\mathbf{3 - 4}$ & $514.05^{\mathrm{a}} \pm 34.74$ & $351.95^{\mathrm{b}} \pm 37.42$ & $466.52^{\mathrm{ab}} \pm 49.97$ & $406.80^{\mathrm{ab}} \pm 35.05$ & $420.36^{\mathrm{ab}} \pm 34.63$ & 0.0419 \\
$\mathbf{4 - 5}$ & $422.47 \pm 48.20$ & $439.50 \pm 59.85$ & $376.33^{2} \pm 67.25$ & $334.61 \pm 47.58$ & $355.75 \pm 31.17$ & NS \\
$\mathbf{0 - 5}$ & $1556.06^{\mathrm{a}} \pm 47.51$ & $1361.93^{\mathrm{b}} \pm 53.46$ & $1257.31^{\mathrm{b}} \pm 39.18$ & $1374.45^{\mathrm{b}} \pm 46.95$ & $1388.89^{\mathrm{b}} \pm 37.72$ & 0.0005 \\
\hline
\end{tabular}

$\mathrm{a}$ and $\mathrm{b}$ Means within the same row with different letters are significantly differed, $\mathrm{C}=\mathrm{Control}$, Cph1=Camphor 1, Cph2=Camphor 2, F1=Fenugreek 1, F2=Fenugreek 2, Pr.= probability, NS = non-significant.

Hind et al (2013) showed that treatment differences only affected dressing \%, breast and abdominal fat. Breast cut reported high weights in group (Cinnamon), (Fenugreek), (Ginger), (control+ antibiotic) (97.4-152.65g) and reported the lowest weight in control $(78.85 \mathrm{~g})$. Estimated dressing percentages in study of (Hind et al 2013) were comparable with the results depicted by (Zomrawi et al 2013) who found $(71.10-73.70 \%)$ is slightly lower than those reported by (Zomrawi et al 2012) who found $(75.15-76.26 \%)$.

\subsubsection{Inedible parts}

Abdominal fat percentage at 5 wk of age recorded highly significant differences between treatments, (Fenugreek 2, Camphor 1 and Fenugreek 1) recorded $(0.83 \%, 1.03 \%$ and $1.07 \%)$ respectively compared to the control (Table 4). Blood percentage showed the lower significant value $(1.37 \%)$ compared to all treatments. It was shown that the percentage of feathers recorded the highest proportion in F2 group (7.03\%) and then less in control $(6.15 \%)$. 
Table 3. Edible parts as affected by Camphor and Fenugreek for Ross broiler hybrid

\begin{tabular}{|c|c|c|c|c|c|c|}
\hline Trait & Control & Cph1 & Cph2 & $\mathbf{F} 1$ & $\mathbf{F 2}$ & Prob. \\
\hline Live body wt., g. & $\begin{array}{c}2119.00^{\mathrm{a}} \\
\pm 37.53\end{array}$ & $\begin{array}{c}1889.75^{c} \\
\pm 18.20\end{array}$ & $\begin{array}{c}1979.25^{\mathrm{bc}} \\
\pm 45.11\end{array}$ & $\begin{array}{c}1996.5^{b c} \\
\pm 47.31\end{array}$ & $\begin{array}{c}2084.3^{a b} \\
\pm 31.88\end{array}$ & 0.0012 \\
\hline Dressing wt. g. & $\begin{array}{c}1552.00^{\mathrm{a}} \\
\pm 33.73\end{array}$ & $\begin{array}{c}1420.50^{b} \\
\pm 19.44\end{array}$ & $\begin{array}{c}1411.08^{b} \\
\pm 33.54\end{array}$ & $\begin{array}{c}1482.7^{a b} \\
\pm 38.62\end{array}$ & $\begin{array}{c}1480.2^{\mathrm{ab}} \\
\pm 41.31\end{array}$ & 0.0477 \\
\hline Dressing wt. \% & $\begin{array}{c}73.20^{\mathrm{ab}} \\
\pm 0.42\end{array}$ & $\begin{array}{l}75.14^{\mathrm{a}} \\
\pm 0.44\end{array}$ & $\begin{array}{l}71.32^{b} \\
\pm 0.33\end{array}$ & $\begin{array}{c}73.47^{\mathrm{ab}} \\
\pm 0.65\end{array}$ & $\begin{array}{l}66.62^{\mathrm{c}} \\
\pm 1.46\end{array}$ & $<0.0001$ \\
\hline Liver wt. g. & $\begin{array}{l}49.40^{\mathrm{a}} \\
\pm 1.18\end{array}$ & $\begin{array}{c}40.12^{\mathrm{bc}} \\
\pm 2.53\end{array}$ & $\begin{array}{l}38.63^{c} \\
\pm 2.62\end{array}$ & $\begin{array}{c}45.19^{\mathrm{ab}} \\
\pm 1.85\end{array}$ & $\begin{array}{c}39.46^{\mathrm{bc}} \\
\pm 0.37\end{array}$ & 0.0042 \\
\hline Liver wt. \% & $\begin{array}{l}\text { 2. } 43^{a b} \\
\pm 0.09\end{array}$ & $\begin{array}{l}2.13^{\mathrm{bc}} \\
\pm 0.11\end{array}$ & $\begin{array}{l}1.99^{c} \\
\pm 0.09\end{array}$ & $\begin{array}{l}2.43^{a} \\
\pm 0.06\end{array}$ & $\begin{array}{l}1.88^{\mathrm{c}} \\
\pm 0.03\end{array}$ & 0.0002 \\
\hline Gizzard wt. g. & $\begin{array}{l}29.27 \\
\pm 0.74\end{array}$ & $\begin{array}{l}26.99 \\
\pm 1.55\end{array}$ & $\begin{array}{l}31.32 \\
\pm 2.86\end{array}$ & $\begin{array}{l}29.57 \\
\pm 1.93\end{array}$ & $\begin{array}{l}27.42 \\
\pm 2.19\end{array}$ & NS \\
\hline Gizzard wt. \% & $\begin{array}{l}1.44^{\mathrm{ab}} \\
\pm 0.02\end{array}$ & $\begin{array}{l}1.37^{\mathrm{b}} \\
\pm 0.09\end{array}$ & $\begin{array}{l}1.73^{a} \\
\pm 0.15\end{array}$ & $\begin{array}{l}1.43^{\mathrm{ab}} \\
\pm 0.09\end{array}$ & $\begin{array}{l}1.23^{b} \\
\pm 0.12\end{array}$ & 0.0388 \\
\hline Heart wt. g. & $\begin{array}{l}9.05^{a} \\
\pm 0.31\end{array}$ & $\begin{array}{l}7.37^{b} \\
\pm 0.25\end{array}$ & $\begin{array}{l}7.47^{b} \\
\pm 0.12\end{array}$ & $\begin{array}{l}8.07^{b} \\
\pm 0.26\end{array}$ & $\begin{array}{l}7.60^{\mathrm{b}} \\
\pm 0.14\end{array}$ & $<0.0001$ \\
\hline Heart wt. \% & $\begin{array}{l}0.42^{a} \\
\pm 0.01\end{array}$ & $\begin{array}{l}0.39^{\mathrm{ab}} \\
\pm 0.01\end{array}$ & $\begin{array}{c}0.39^{\mathrm{ab}} \\
\pm 0.009\end{array}$ & $\begin{array}{l}0.41^{\mathrm{ab}} \\
\pm 0.01\end{array}$ & $\begin{array}{c}0.37^{b} \\
\pm 0.006\end{array}$ & 0.0154 \\
\hline Giblets wt. g. & $\begin{array}{l}89.07^{a} \\
\pm 1.02\end{array}$ & $\begin{array}{l}74.50^{\mathrm{b}} \\
\pm 2.53\end{array}$ & $\begin{array}{l}77.43^{b} \\
\pm 5.01\end{array}$ & $\begin{array}{c}82.84^{\mathrm{ab}} \\
\pm 2.51\end{array}$ & $\begin{array}{l}74.49^{b} \\
\pm 2.22\end{array}$ & 0.0184 \\
\hline Giblets wt.\% & $\begin{array}{l}4.20^{a} \\
\pm 0.13\end{array}$ & $\begin{array}{l}3.90^{a} \\
\pm 0.12\end{array}$ & $\begin{array}{l}4.12^{\mathrm{a}} \\
\pm 0.21\end{array}$ & $\begin{array}{r}4.27^{a} \\
\pm 0.09\end{array}$ & $\begin{array}{l}3.43^{b} \\
\pm 0.19\end{array}$ & 0.0048 \\
\hline Minor pectoralis wt. g. & $\begin{array}{l}45.28 \\
\pm 2.18\end{array}$ & $\begin{array}{l}42.16 \\
\pm 2.39\end{array}$ & $\begin{array}{l}44.81 \\
\pm 6.77\end{array}$ & $\begin{array}{r}40.99 \\
\pm 1.03\end{array}$ & $\begin{array}{l}40.99 \\
\pm 2.21\end{array}$ & NS \\
\hline Minor pectoralis wt. \% & $\begin{array}{c}2.23 \\
\pm 0.07\end{array}$ & $\begin{array}{c}2.01 \\
\pm 0.05\end{array}$ & $\begin{array}{c}2.44 \\
\pm 0.44\end{array}$ & $\begin{array}{r}2.10 \\
\pm 0.04\end{array}$ & $\begin{array}{r}1.78 \\
\pm 0.05\end{array}$ & NS \\
\hline Major pectoralis wt. g. & $\begin{array}{c}191.12^{\mathrm{a}} \\
\pm 7.84\end{array}$ & $\begin{array}{c}168.26^{\mathrm{b}} \\
\pm 4.87\end{array}$ & $\begin{array}{c}173.81^{\mathrm{ab}} \\
\pm 7.13\end{array}$ & $\begin{array}{c}179.83^{a b} \\
\pm 8.11\end{array}$ & $\begin{array}{c}185.28^{\mathrm{ab}} \\
\pm 5.63\end{array}$ & 0.0195 \\
\hline Major pectoralis wt. \% & $\begin{array}{c}8.98 \\
\pm 0.20\end{array}$ & $\begin{array}{c}8.56 \\
\pm 0.20\end{array}$ & $\begin{array}{c}9.18 \\
\pm 0.20\end{array}$ & $\begin{array}{c}8.55 \\
\pm 0.25\end{array}$ & $\begin{array}{c}8.48 \\
\pm 0.28\end{array}$ & NS \\
\hline Drum muscle wt. g. & $\begin{array}{l}91.08^{\mathrm{a}} \\
\pm 2.35\end{array}$ & $\begin{array}{l}93.99^{a} \\
\pm 1.55\end{array}$ & $\begin{array}{l}81.21^{b} \\
\pm 2.25\end{array}$ & $\begin{array}{l}94.38^{\mathrm{a}} \\
\pm 2.89\end{array}$ & $\begin{array}{l}89.56^{a} \\
\pm 2.46\end{array}$ & 0.0008 \\
\hline Drum muscle wt. \% & $\begin{array}{l}4.29^{\mathrm{b}} \\
\pm 0.06\end{array}$ & $\begin{array}{l}5.05^{a} \\
\pm 0.05\end{array}$ & $\begin{array}{l}4.16^{\mathrm{b}} \\
\pm 0.09\end{array}$ & $\begin{array}{l}4.80^{\mathrm{a}} \\
\pm 0.11\end{array}$ & $\begin{array}{l}4.21^{\mathrm{b}} \\
\pm 0.15\end{array}$ & $<0.0001$ \\
\hline Thigh muscle wt. g. & $\begin{array}{c}188.36^{a} \\
\pm 6.61\end{array}$ & $\begin{array}{c}157.45^{b} \\
\pm 5.68\end{array}$ & $\begin{array}{c}166.16^{\mathrm{b}} \\
\pm 7.76\end{array}$ & $\begin{array}{c}167.77^{\mathrm{b}} \\
\pm 4.24\end{array}$ & $\begin{array}{c}136.12^{c} \\
\pm 7.07\end{array}$ & 0.0003 \\
\hline Thigh muscle wt. \% & $\begin{array}{l}8.87^{a} \\
\pm 0.15\end{array}$ & $\begin{array}{l}8.21^{a} \\
\pm 0.33\end{array}$ & $\begin{array}{l}8.25^{a} \\
\pm 0.41\end{array}$ & $\begin{array}{l}8.13^{a} \\
\pm 0.10\end{array}$ & $\begin{array}{r}6.55^{\mathrm{b}} \\
\pm 0.36\end{array}$ & 0.0004 \\
\hline
\end{tabular}

$\mathrm{a}$ and $\mathrm{b}$ Means within the same row with different letters are significantly differed, $\mathrm{C}=\mathrm{Control}$, Cph1=Camphor 1, Cph2=Camphor 2, F1=Fenugreek 1, F2=Fenugreek 2, Pr.= probability, NS = non-significant 
Table 4. Inedible parts as affected by Camphor and Fenugreek traits for Ross broiler hybrid

\begin{tabular}{|l|c|c|c|c|c|c|}
\hline \multicolumn{1}{|c|}{ Trait } & Control & Cph1 & Cph2 & F1 & F2 & Prob. \\
\hline Live body wt., g. & $2119.00^{\mathrm{a}}$ & $1889.75^{\mathrm{c}}$ & $1979.25^{\mathrm{bc}}$ & $1996.50^{\mathrm{bc}}$ & $2084.33^{\mathrm{ab}}$ & 0.0012 \\
& \pm 37.53 & \pm 18.20 & \pm 45.11 & \pm 47.31 & \pm 31.88 & \\
Abdominal fat wt. g. & $29.72^{\mathrm{a}}$ & $20.19^{\mathrm{b}}$ & $22.19^{\mathrm{b}}$ & $18.58^{\mathrm{b}}$ & $18.62^{\mathrm{b}}$ & 0.0044 \\
& \pm 2.97 & \pm 1.20 & \pm 2.22 & \pm 2.59 & \pm 1.48 & \\
Abdominal fat wt. \% & $1.43^{\mathrm{a}}$ & $1.03^{\mathrm{b}}$ & $1.19^{\mathrm{ab}}$ & $1.07^{\mathrm{b}}$ & $0.83^{\mathrm{b}}$ & 0.0187 \\
& \pm 0.15 & \pm 0.07 & \pm 0.10 & \pm 0.15 & \pm 0.08 & \\
Blood wt. g. & $42.00^{\mathrm{b}}$ & $69.33^{\mathrm{a}}$ & $73.75^{\mathrm{a}}$ & $70.50^{\mathrm{a}}$ & $57.00^{\mathrm{ab}}$ & 0.024 \\
& \pm 6.50 & \pm 12.25 & \pm 8.20 & \pm 2.63 & \pm 5.06 & \\
Blood wt. \% & $1.37^{\mathrm{b}}$ & $3.66^{\mathrm{a}}$ & $3.65^{\mathrm{a}}$ & $3.52^{\mathrm{a}}$ & $2.77^{\mathrm{a}}$ & 0.0007 \\
& \pm 0.06 & \pm 0.66 & \pm 0.32 & \pm 0.05 & \pm 0.25 & \\
Feathers wt. g. & $130.66^{\mathrm{ab}}$ & $118.75^{\mathrm{b}}$ & $148.25^{\mathrm{a}}$ & $153.00^{\mathrm{a}}$ & $145.00^{\mathrm{ab}}$ & 0.0707 \\
& \pm 13.54 & \pm 5.22 & \pm 9.90 & \pm 10.51 & \pm 7.98 & \\
Feathers wt. \% & $6.15^{\mathrm{b}}$ & $6.31^{\mathrm{ab}}$ & $7.46^{\mathrm{ab}}$ & $7.68^{\mathrm{a}}$ & $7.03^{\mathrm{ab}}$ & 0.096 \\
\hline
\end{tabular}

$\mathrm{a}$ and $\mathrm{b}$ Means within the same row with different letters are significantly differed, $\mathrm{C}=\mathrm{C}$ Control, $\mathrm{Cph} 1=\mathrm{Camphor} 1$, Cph2=Camphor 2, F1=Fenugreek 1, F2=Fenugreek 2, Pr.= probability, NS = non-significant

For abdominal fat, only group (control+ antibiotic) and (Fenugreek) were significantly different from each other, while (control), (Cumin), (Ginger) and $F$ (Cinnamon) were not different from group (control+ antibiotic) or (Fenugreek). These findings agreed with (Zhang et al 2009 and Javed et al 2009) who observed that dressing percentage, breast weight and leg weight increased significantly when certain spices were added to the ration.

\section{Immunocompetence measurements}

In this study, Table (5) showed that the highest value for spleen percentage was recorded in $\mathrm{F} 1$ group $(0.12 \%)$ and the lowest value showed in control and Cph2 (0.08\%). Rabia (2010) concluded that there is an insignificant difference for spleen weight percentage between control group $(0.20 \%)$ and the group which fed on (3 $\mathrm{g}$ fenugreek $/ \mathrm{kg}$ diet) $(0.19 \%)$ for broiler at 42 days of age.

Bursa gland recorded the highest value for F2 group $(0.06 \%)$ and the lowest Value was recorded by Control and Cph2 group (0.02\%), followed by control $(0.02 \%)$. Rabia (2010) concluded that there is an insignificant difference for bursa of fabriciuos weight percentage between control group $(0.14 \%)$ and the group which fed on $3 \mathrm{~g}$ fenugreek $/ \mathrm{kg}$ (0.11\%). Khadr and Abdel-Fattah (2007) showed that the bursa weight was not affected by addition of fenugreek with ( $1 \%$ and $2 \%$ ) levels compared to the control.
Thymus gland showed significant differences between traits. The F1 group recorded the highest value $(0.12 \%)$ and the lowest value showed in Cph2 group (0.02\%). Khadr and Abdel-Fattah (2007) showed that fenugreek seed at level of $1 \%$ increased thymus weight which was higher than control and the $2 \%$ treatment.

Hind et al (2013) reported that the weight of the immunological organs (thymus and spleen) was not significantly affected by the treatment differences, while the third organ, bursa weight was significantly affected by treatment differences. (Cumin) and (ginger) showed the highest bursa weight (2.72 and $1.84 \mathrm{~g}$ ), respectively, while (control), (control+ antibiotic), (Fenugreek), (Cinnamon) were not significantly different. On the other hand, (Bin et al 2003) reported that the addition of Fenugreek to boiler feeds lead to increased bursa weight.

Injection of phytohemagglutinin-P at a selected site in chickens considered as an inducer of localized in vivo lymphoproliferative response (Cheema et al 2003). The response was measured in this study at 24, 48 and $72 \mathrm{hr}$ post PHA-P injection into the toe-web and the results showed in Table (6). At $24 \mathrm{hr}$ after injection chickens which fed diet with Camphor 2 and Fenugreek 2 addition had the maximum cutaneous basophilic hypersensitivity swelling response with insignificantly difference compared to the other treatments and control. Swelling response decreased by 48 and 72 hour. 
Table 5. lymphoid organs as affected by Camphor and Fenugreek treatments for Ross broiler hybrid

\begin{tabular}{|l|c|c|c|c|c|c|}
\hline \multicolumn{1}{|c|}{ Trait } & Control & Cph1 & Cph2 & F1 & F2 & Prob. \\
\hline Spleen wt (g.) & $1.82^{\mathrm{b}} \pm 0.20$ & $1.89^{\mathrm{b}} \pm 0.14$ & $1.57^{\mathrm{b}} \pm 0.14$ & $2.45^{\mathrm{a}} \pm 0.21$ & $2.01^{\mathrm{ab}} \pm 0.12$ & 0.0071 \\
Spleen wt \% & $0.08^{\mathrm{b}} \pm 0.008$ & $0.10^{\mathrm{ab}} \pm 0.007$ & $0.08^{\mathrm{b}} \pm 0.009$ & $0.12^{\mathrm{a}} \pm 0.01$ & $0.10^{\mathrm{ab}} \pm 0.006$ & 0.0159 \\
Bursa wt (g.) & $0.61^{\mathrm{b}} \pm 0.07$ & $0.62^{\mathrm{b}} \pm 0.05$ & $0.52^{\mathrm{b}} \pm 0.01$ & $0.89^{\mathrm{ab}} \pm 0.09$ & $1.18^{\mathrm{a}} \pm 0.23$ & 0.0023 \\
Bursa wt \% & $0.02^{\mathrm{b}} \pm 0.002$ & $0.03^{\mathrm{b}} \pm 0.002$ & $0.02^{\mathrm{b}} \pm 0.001$ & $0.04^{\mathrm{ab}} \pm 0.005$ & $0.06^{\mathrm{a}} \pm 0.01$ & 0.0028 \\
Thymus wt (g.) & $2.45^{\mathrm{a}} \pm 0.36$ & $1.98^{\mathrm{ab}} \pm 0.25$ & $1.47 \pm 0.12$ & $2.45^{\mathrm{a}} \pm 0.25$ & $1.89^{\mathrm{ab}} \pm 0.36$ & 0.0063 \\
Thymus wt \% & $0.11^{\mathrm{ab}} \pm 0.01$ & $0.10^{\mathrm{ab}} \pm 0.01$ & $0.07^{\mathrm{b}} \pm 0.004$ & $0.12^{\mathrm{a}} \pm 0.01$ & $0.09^{\mathrm{ab}} \pm 0.01$ & 0.0086 \\
\hline
\end{tabular}

$\mathrm{a}$ and $\mathrm{b}$ Means within the same row with different letters are significantly differed, $\mathrm{C}=\mathrm{Control}$, Cph1=Camphor 1, Cph2=Camphor 2, F1=Fenugreek 1, F2=Fenugreek 2, Pr.= probability, NS = non-significant

Table 6. Toe web dermal swelling response (difference) to phytohemagglutinin-P injection as affected by Camphor and Fenugreek treatments for Ross broiler hybrid

\begin{tabular}{|c|c|c|c|c|c|c|}
\hline \multirow{2}{*}{$\begin{array}{c}\text { Time } \\
\text { (hr.) }\end{array}$} & \multicolumn{5}{|c|}{ Trait } & \multirow{2}{*}{ Prob. } \\
\cline { 2 - 7 } & \multicolumn{5}{|c|}{ Cph1 } & \multicolumn{2}{|c|}{ Cph2 } & F1 & F2 & \\
\hline 24 hr. & $0.0188 \pm 0.0064$ & $0.0277 \pm 0.0045$ & $0.0295 \pm 0.0049$ & $0.0258 \pm 0.0054$ & $0.0303 \pm 0.0036$ & NS \\
48 hr. & $0.0097^{\mathrm{b}} \pm 0.0027$ & $0.0140^{\mathrm{ab}} \pm 0.0045$ & $0.0258^{\mathrm{a}} \pm 0.0075$ & $0.0125^{\mathrm{ab}} \pm 0.0040$ & $0.0115^{\mathrm{ab}} \pm 0.0037$ & 0.0163 \\
72 hr. & $0.0117 \pm 0.0045$ & $0.0115 \pm 0.0037$ & $0.0066 \pm 0.0028$ & $0.0051 \pm 0.0047$ & $0.0100 \pm 0.0028$ & NS \\
\hline
\end{tabular}

$\mathrm{a}$ and $\mathrm{b}$ Means within the same row with different letters are significantly differed, $\mathrm{C}=\mathrm{Control}$, Cph1=Camphor 1, Cph2=Camphor 2, F1=Fenugreek 1, F2=Fenugreek 2, Pr.= probability, NS = non-significant

The Cph2 treatment had significantly higher dermal swelling response at $48 \mathrm{hr}$ compared to another treatments and control group. Motamedi and Taklimi (2014) reported that the treatment containing $0.5 \%$ fenugreek had the maximum value of lymphocytes percentage (69.00) and control treatment had the minimum value (67.75).

Sheep red blood cells (SRBCs) have been chosen in this study as a non-pathogenic antigen for virus. Total anti-SRBCs antibody presented in (Table 7) speculated that the birds which fed on F1 and F2 groups had significantly hyper responder to SRBCs at 7 days post-secondary injection, while at 14 days post-secondary injection Cph2 and F2 groups had significantly hyper responses to SRBCs. Motamedi and Taklimi (2014) reported that the mean SRBC in the group of $0.5 \%$ fenugreek has a significant difference with the control group. They showed that there is no significant difference between treatments, but $1 \%$ fenugreek group had an increase compared to the control group.

With respect to Immunoglobulin-M, results showed that F1 and F2 groups had significantly hyper responder to SRBCs at 7 days postsecondary injection, while at 14 days postsecondary injection F2 group had significantly hyper response to SRBCs compared to the another groups. Motamedi and Taklimi (2014) reported that the effect of various treatments did not become significant on the data of immunoglobulin $\mathrm{M}$. the control treatment and $0.5 \%$ fenugreek had the 
Table 7. Total anti-SRBCs antibody, immunoglobulin-M and immunoglobulin-G of Ross broiler hybrids affected by Camphor and Fenugreek treatments

\begin{tabular}{|c|c|c|c|c|}
\hline \multicolumn{5}{|c|}{ Total anti-SRBCs antibody titer } \\
\hline Trait & 7PPI & 14PPI & 7PSI & 14PSI \\
\hline C & $4.66 \pm 1.20$ & $3.00 \pm 0.40$ & $7.50^{\mathrm{ab}} \pm 1.20$ & $2.00^{b} \pm 0.40$ \\
\hline Cph1 & $4.50 \pm 0.50$ & $2.50 \pm 0.65$ & $6.75^{\mathrm{ab}} \pm 0.85$ & $2.25^{b} \pm 0.47$ \\
\hline Cph2 & $3.33 \pm 2.33$ & $2.75 \pm 0.25$ & $4.75^{\mathrm{b}} \pm 0.85$ & $4.75^{\mathrm{a}} \pm 0.62$ \\
\hline F1 & $4.33 \pm 0.66$ & $2.25 \pm 0.62$ & $9.00^{a} \pm 1.00$ & $2.25^{b} \pm 0.25$ \\
\hline F2 & $4.00 \pm 0.00$ & $2.75 \pm 0.25$ & $9.00^{\mathrm{a}} \pm 1.08$ & $5.00^{\mathrm{a}} \pm 0.70$ \\
\hline Prob. & NS & NS & 0.009 & 0.001 \\
\hline \multicolumn{5}{|c|}{ Immunoglobulin-M } \\
\hline Trait & 7PPI & 14PPI & 7PSI & 14PSI \\
\hline C & $1.66 \pm 1.20$ & $2.25 \pm 0.25$ & $5.25^{a b} \pm 1.31$ & $1.25^{\mathrm{b}} \pm 0.25$ \\
\hline Cph1 & $1.25 \pm 0.75$ & $1.00 \pm 0.57$ & $4.25^{\mathrm{ab}} \pm 1.08$ & $1.25^{\mathrm{b}} \pm 0.47$ \\
\hline Cph2 & $2.00 \pm 3.00$ & $2.00 \pm 0.00$ & $2.00^{\mathrm{b}} \pm 0.00$ & $1.66^{b} \pm 0.33$ \\
\hline $\mathbf{F} 1$ & $1.33 \pm 0.66$ & $1.25 \pm 0.47$ & $5.66^{a b} \pm 0.66$ & $1.25^{b} \pm 0.47$ \\
\hline $\mathbf{F 2}$ & $0.25 \pm 0.25$ & $1.50 \pm 0.65$ & $7.00^{a} \pm 0.91$ & $3.50^{\mathrm{a}} \pm 0.65$ \\
\hline Prob. & NS & NS & 0.004 & 0.003 \\
\hline \multicolumn{5}{|c|}{ Immunoglobulin-G } \\
\hline Trait & 7PPI & 14PPI & 7PSI & 14PSI \\
\hline C & $3.00 \mathrm{a} \pm 0.00$ & $1.00 \pm 0.00$ & $3.00^{\mathrm{ab}} \pm 0.00$ & $1.75^{\mathrm{b}} \pm 0.00$ \\
\hline Cph1 & $2.00 b \pm 0.00$ & $1.50 \pm 0.28$ & $3.33^{a b} \pm 0.88$ & $1.33^{b} \pm 0.33$ \\
\hline Cph2 & $2.00 b \pm 0.00$ & $1.00 \pm 0.00$ & $4.50^{\mathrm{a}} \pm 0.50$ & $3.75^{\mathrm{a}} \pm 0.25$ \\
\hline $\mathbf{F 1}$ & $3.00 a \pm 0.00$ & $1.33 \pm 0.33$ & $3.33^{a b} \pm 0.33$ & $1.33^{b} \pm 0.33$ \\
\hline F2 & $3.50 a \pm 0.50$ & $1.66 \pm 0.33$ & $2.66^{b} \pm 0.33$ & $2.00^{b} \pm 0.00$ \\
\hline Prob. & 0.006 & NS & 0.04 & 0.001 \\
\hline
\end{tabular}

minimum value (1.48). Concerning to Immunoglobulin-G, F2 group showed a significantly hyper responder to SRBCs at 7 days post primary injection, while Cph2 group showed a significantly hyper responder to SRBCs at 7 and 14 days postsecondary injection. Motamedi and Taklimi (2014) reported that the effect of various treatments has been significant on immunoglobulin $G$ so that, the mean of group containing $1 \%$ fenugreek have a significant difference. Also, there is no significant difference between treatment containing $1 \%$ fenugreek and control group.

\section{CONCLUSION}

Considering the results obtained from the current study it could be concluded that the fenugreek seeds powder and camphor leaves powder may increase body weight, with additive $(1.5 \mathrm{~g} / 1 \mathrm{~kg}$ diet) carcass characteristics and also improving the cellular and humeral immunity for broiler chickens under environmental conditions of Egypt.

\section{REFERENCES}

Bach K.K.E. 2001. Development of antibiotic resistance and options to replace antimicrobials in animal diets. Proceedings of the Nut. Society, 60, 291-299.

Barton M. 2000. Antibiotic use in animal feed its impact on human health. Nut Research Reviews, 13, 299-279.

Basch E., Ulbricht C., Kuo G., Szapary P. and Smith M. 2003. Therapeutic applications of 

response in broiler chicken

fenugreek. Alternative Medicine Review, 8, 20-27.

Bin-afeez B., Haque R., Rarvez S., Pandey S., Sayeed I. and Raisuddin S. 2003. Immunomudulatory effects of fenugreek (Trigonella foenum graceum) extract in mice. International Immunopharmacology 3, 257-265.

Cheema M.A., Qureshi M.A. and Havenstein G.B. 2003. A comparison of the immune response of a 2001 commercial broiler with a 1957 random bred broiler strain when fed representative 1957 and 2001 broiler diets. Poultry Sci., 82, 1519-1529.

Duncan D.B. 1955. Multiple range and multiple $F$ tests. Biometrics, 11(1), 1-42.

Ellenhorn M.J. and Barceloux D.G. 1998. Camphor. In Medical Technology: Diagnosis and treatment of human poisoning. New York: Elsevier, USA, pp. 505-507.

Farman Ullah K, Durrani F.R., Asad S, Rifat Ullah K. and Shabana N. 2009. Effects of Fenugreek (Trigonellafoenum-graecum) seed extract onvisceral organs of broiler chicks. ARPN J. of Agric. and Biological Sci., 4(1), 58-60.

Gibson D.E., Moore G.P. and Pfaff J.A. 1989. Camphor ingestion. Am. J. Emerg. Med. 7, 4143.

Hind A.A. Elagib, Saadia A. Abbas and Khalid M. Elamin 2013. Effect of Different Natural Feed additives Compared to Antibiotic on Performance of Broiler Chicks under High Temperature. Bull. Env. Pharmacol. Life Sci., 2(11), 139-144.

Javed M., Durrani F., Hafeez A., Khan R.U. and Ahmad I. 2009. Effect of aqueous extract of plant mixture on carcass quality of broiler chicks. ARPN J. of Agric. and Biological Sci., 4, 37-40.

Khadr N.A. and Abdel-Fattah F.A.I. 2007. Response of broiler chickens to diet containing fenugreek seed (Trigonellafoenum-graecum L.) as a natural feed additive._Benha Veterinary Medical J. 18, 27-50.

Mamoun Tariq, Mukhtar Ahmed Mukhtar and Mohamed H.T. abidi 2014. Effect of Fenugreek Seed on the Performance, Carcass and Some Blood Attributes. Adv. Res Agric. Vet Sci. 1(1), 6-11.
Mazur W.M., Duke J.A., Wahala K., Rasku S. and Adlercreutz H. 1998. Isoflavonoids and lignins in legumes: Nutritional and health aspects in human, J. Nutr. Biochem., 9, 193200.

Motamedia S.M. and Taklimib S.M.M. 2014. Investigating the effect of fenugreek seed powder and garlic powder in the diet on immune response of commercial laying hens' egg. Indian J. Sci. Res. 3(1), 277-283.

Rabia J. Abbas 2010. Effect of Using Fenugreek, Parsley and Sweet Basil Seeds as Feed Additives on the Performance of Broiler Chickens. Int. J. of Poultry Sci., 9(3), 278-282.

SAS Institute Inc. 2004. SAS ISHARE ${ }^{\circledR} 9.1$ User's Guide, Cary, NC: SAS Institute Inc.

Srinivasan K. 2006. Fenugreek (Trigonellafoenum-graecum): A Review of Health Beneficial Physiological Effects. Food Reviews Int. 22(2), 203-224.

Weerasingha A.S. and Atapattu N.S.B.M. 2013. Effects of Fenugreek (Trigonellafoenumgraecum L.) Seed Powder on Growth Performance, Visceral Organ Weight, Serum Cholesterol Levels and the Nitrogen Retention of Broiler Chicken. Tropical Agric. Res. 24(3), 289-295.

Yamamoto Y. and Glick B. 1982. A comparison of the immune response between two lines of chickens selected for differences in the weight of the bursa of Fabricius. Poultry Sci., 61, 2129-2132.

Zhang G.F., Yang Z.B., Wang Y., Yang W.R., Jiang S.Z. and Gai G.S. 2009. Effects of ginger root (Zingiber officinale) processed to different particle sizes on growth performance, antioxidant status, and serum metabolites of broiler chickens. Poultry Sci., 88(10), 21592166.

Zomrawi W.B., Abdel Atti K.A.A., Dousa B.M. and Mahala A.G. 2012. The effect of ginger root powder (Zinger officinale) supplementation on broiler chicks performance, blood and serum constituents. Online J. of Animal and Feed Research, 6, 457-460.

Zomrawi W.B., Abdel Atti K.A.A., Dousa B.M. and Mahala A.G. 2013. The effect of dietary ginger root powder (Zingiber officinale) on broiler chicks' performance, carcass characteristics and serum constituents. J. of Animal Sci. Advances, 3, 42-47. 


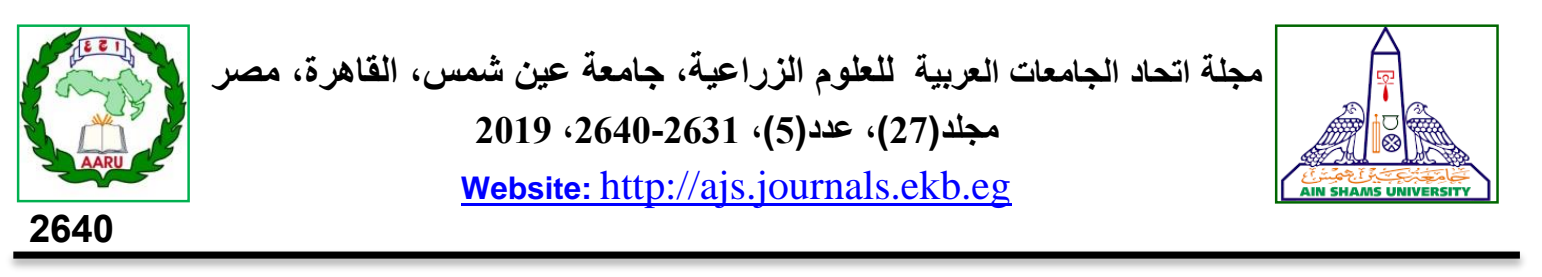

تأثير إضافة محفزات النمو علي الأداء الانتاجي والاستجابة المناعية لاجاج اللحم

[210]

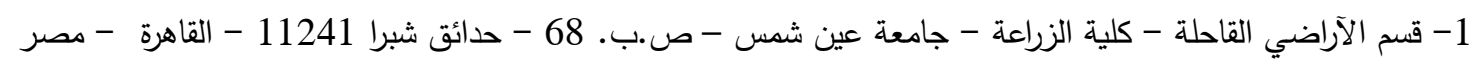

$$
\begin{aligned}
& \text { 2- قسم البساتين - كلية الزراعة - جامعة عين شمس - ص.ب. } \\
& \text { 2- قسم الدواجن - كلية الزراعة - جامعة عين شمس - ص.ب. } 68 \text { - حدائق شبرا } 11241 \text { - القاهرة - مصر }
\end{aligned}
$$

*Corresponding author: mohamedhashem10@icoud.com

Received 11 September, 2019

Accepted 12 January, 2019

حلبة 2 أقل قيمة للنسبة المئوية للحوائج مقارنة ببقية

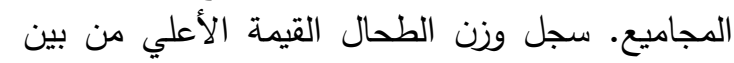

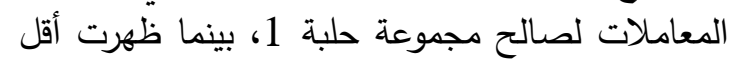

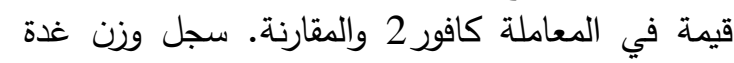

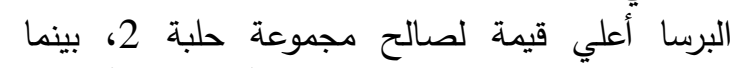
سجلت بؤمجموعة المقارنة وكافور 2 أقل قيمة. أظهرت البهاح

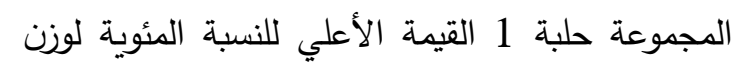

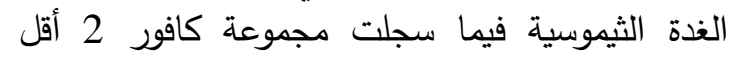
قيمة. أظهرت مجاميع حلبة 2 وكافور 2 استجابة

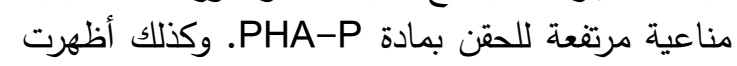

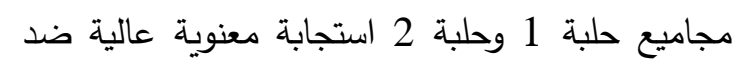
خلايا دم الغنم الحمراء عند اليوم السابع بعد الحقن

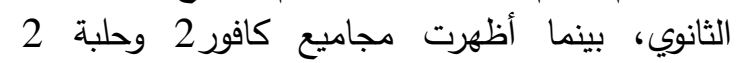

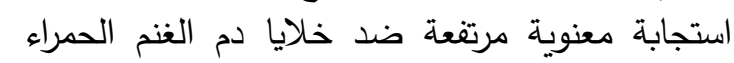

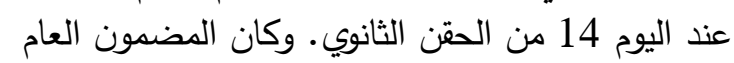

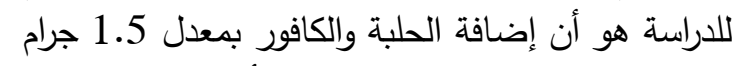
لكل 1 كجم عليقة هي النسبة الأفضل خلافل هذه الداء الدراسة لتحقيق آداء أُفضل تحت النية الظروف البيئية

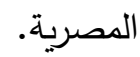

الكلمات الدالة: الحلبة، الكافور، الإنتاج، المناعه، دجاج اللحم

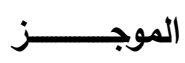

الغرض من هذه الدراسة هو معرفة تأثير إضافة

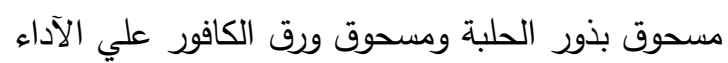

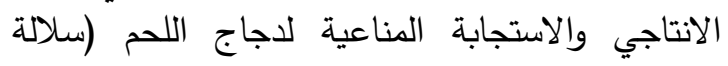

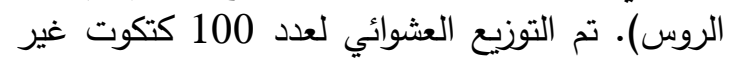

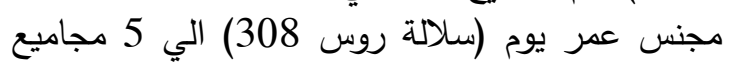
ولكل معاملة مكررتان، داخل كل مكررة 10 كتاكيت تم

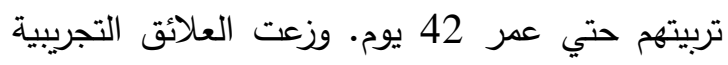
كالتالي: عليقة مقارنة، عليقة مزودة بمسحوق بذور

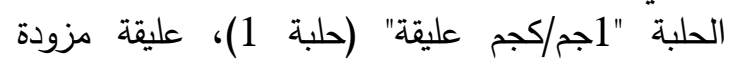
بمسحوق بذور الحلبة "1.5جم/كجم عليقة" (حلبة2)،

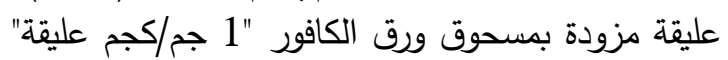
(كافور 1) وعليقة مزودة بمسحوق ورق الكافور ل1.

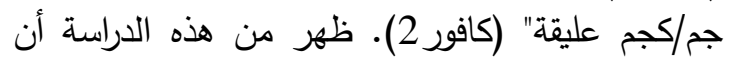

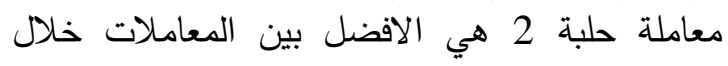

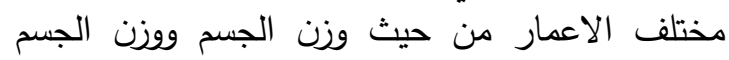

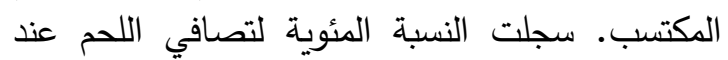
عمر 5 أسابيع أعلي زيادة معنوية لمجموعة كافور المبة 1.

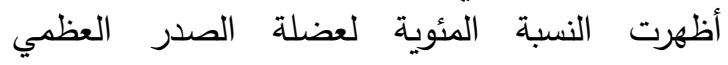
والصغري زيادة طفيفة غير معنوية لمعاملة كافور2. وسجلت معاملة كافور 1 قيمة مرتفعة معنوية لمنية للنسبة المئوية لوزن عضلة الدبوس. بينما سجلت مجموعة 